EPJ Web of Conferences 4, 03001 (2010)

DOI:10.1051/epjconf/20100403001

C) Owned by the authors, published by EDP Sciences, 2010

\title{
Combination and interpretation of observables in Cosmology
}

\author{
Jean-Marc VIREY \\ Centre de Physique Théorique, Marseille
}

\begin{abstract}
The standard cosmological model has deep theoretical foundations but need the introduction of two major unknown components, dark matter and dark energy, to be in agreement with various observations. Dark matter describes a non-relativistic collisionless fluid of (non baryonic) matter which amount to $25 \%$ of the total density of the universe. Dark energy is a new kind of fluid not of matter type, representing $70 \%$ of the total density which should explain the recent acceleration of the expansion of the universe.

Alternatively, one can reject this idea of adding one or two new components but argue that the equations used to make the interpretation should be modified on cosmological scales. Instead of dark matter one can invoke a failure of Newton's laws. Instead of dark energy, two approaches are proposed : general relativity (in term of the Einstein equation) should be modified, or the cosmological principle which fixes the metric used for cosmology should be abandonned.

One of the main objective of the community is to find the path of the relevant interpretations thanks to the next generation of experiments which should provide large statistics of observationnal data. Unfortunately, cosmological informations are difficult to pin down directly from the measurements, and it is mandatory to combine the various observables to get the cosmological parameters. This is not problematic from the statistical point of view, but assumptions and approximations made for the analysis may bias our interprettion of the data. Consequently, a strong attention should be paied to the statistical methods used to make parameters estimation and for model testing.
\end{abstract}

After a review of the basics of cosmology where the cosmological parameters are introduced, we discuss the various cosmological probes and their associated observables used to extract cosmological informations. We present the results obtained from several statistical analyses combining data of different nature but we insist on the main drawbacks that can falsify our final interpretation.

This is an Open Access article distributed under the terms of the Creative Commons Attribution-Noncommercial License 3.0, which permits unrestricted use, distribution, and reproduction in any noncommercial medium, provided the original work is properly cited. 